Article

\title{
Early Lesion of Post-Primary Tuberculosis: Subclinical Driver of Disease and Target for Vaccines and Host-directed Therapies.
}

\author{
Robert E. Brown ${ }^{1}$ and Robert L. Hunter ${ }^{*}$ \\ 1 Department of Pathology and Laboratory Medicine, University of Texas Health Sciences Center at Houston, \\ Houston, TX 77030, USA: robert.brown@uth.tmc.edu, Robert.L.Hunter@uth.tmc.edu \\ * Correspondence: Correspondence: Robert.L.Hunter@uth.tmc.edu Tel: 0017138980666
}

\begin{abstract}
The characteristic lesion of primary tuberculosis is the granuloma as is widely studied in human tissues and animal models. Post-primary tuberculosis is different. It develops only in human lungs and begins as a prolonged subclinical obstructive lobular pneumonia that slowly accumulates mycobacterial antigens and host lipids in alveolar macrophages with nearby highly sensitized $\mathrm{T}$ cells. After several months, the lesions undergo necrosis to produce a mass of caseous pneumonia large enough to fragment and be coughed out to produce a cavity or be retained as the focus of a postprimary granuloma. Here we extend these findings with the demonstration of mycobacterial antigen, but not AFB, of M. tuberculosis in the cytoplasm of ciliated bronchiolar epithelium and alveolar pneumocytes in association with elements of the programmed death ligand 1 (PD-L1), cyclooxygenase (COX)-2, and fatty acid synthase (FAS) pathways in the early lesion. This suggests that M. tuberculosis uses its secreted antigens to coordinate prolonged subclinical development of the early lesions in preparation for a necrotizing reaction sufficient to produce a cavity, post-primary granulomas and fibrocaseous disease.
\end{abstract}

Keywords. Tuberculosis, secretory antigens, bronchiolar epithelium, alveolar pneumocytes, M2 polarization, COX-2, FAS, Pathogenesis, early lesion

\section{Introduction}

Pathologists in the pre antibiotic era conducted many autopsies and so were familiar with a series of tuberculous lesions that are seldom seen today [1]. They recognized that the onset of clinical post-primary tuberculosis (PPTB) is preceded by 1-2 years of asymptomatic subclinical development of the early lesion in the lung before appearance of caseous pneumonia that leads to cavities, post-primary granulomas and fibrocaseous disease [2-4]. Today, since there is no medical reason to biopsy the early lesions and autopsies are infrequent, most investigators are unaware of their existence even though they remain familiar to radiologists as the 'tree-in-bud' sign characteristic of developing PPTB [5].

The early lesions of PPTB do not begin until after establishment of sufficient immunity to prevent disease in all extra pulmonary and most pulmonary sites [1]. PPTB begins as an alveolitis and spreads for months via bronchi as an obstructive lobular pneumonia that is recognizable on high resolution CT scans as the 'tree-in-bud' sign [5]. The early lesions are paucibacillary and consist of alveolar macrophages that slowly accumulate secreted mycobacterial antigens and host lipids as they become foamy and accumulate behind obstructed bronchi in close association with highly sensitized T cells [6,7]. Many lesions spontaneously regress, but some undergo necrosis to produce caseous pneumonia that is either coughed out to form cavities or is retained to become the focus of post-primary granulomas and fibrocaseous disease [6]. Once a cavity is formed, masses of bacteria accumulate on its surface where they can be coughed out into the environment to infect new hosts. 
Post-primary granulomas are easily identified because they contain ghosts of alveoli rather than the homogeneous caseum of primary granulomas [1]. Primary and post-primary granulomas are seldom found together in the same lung. We reported sequestration of secreted mycobacterial antigens in foamy alveolar macrophages in the developing lesions of post-primary $\mathrm{TB}$ and that they are released with the onset of caseation necrosis in a fashion suggestive of the Koch phenomenon [6].

Here we report finding M. tuberculosis (MTB) antigens, but not acid-fast bacilli (AFB), in alveolar lining cells (pneumocytes) and ciliated bronchial cells together with elements of the programmed death ligand 1 (PD-L1), cyclo-oxygenase (COX)-2, and fatty acid synthase (FAS) pathways in developing lesions of PPTB. This suggests that MTB use secreted antigens to orchestrate an extended sequence of subclinical lesions that prepare for a sudden massive necrotizing pneumonia sufficient to produce cavities from which the organisms can escape to new hosts. Post-primary granulomas arise to surround foci of caseous pneumonia that are not coughed out to form cavities. They persist to become fibrocaseous disease. Most nascent lesions regress spontaneously leaving apical scars. If we understood why they regress, it might be possible to make them all regress and thereby drive MTB to extinction.

\section{Results}

Histologic slides from over 50 autopsies of people who died of untreated TB were examined to identify tissue sections with the early lesion of PPTB as previously described [1, 7]. Briefly, the early lesions are an obstructive lobular pneumonia that can be distinguished from non-specific inflammation by the presence of mycobacterial antigens in foamy alveolar macrophages. This disease occurs preferentially in immunocompetent young adults with strong tuberculin skin tests [2-4]. Since all of the patients were adults who died of pulmonary $\mathrm{TB}$, they all had advanced post-primary lesions with little or no extra pulmonary TB. However, since the lesions of PPTB develop independently of one another, one commonly finds early lesions in the same lung as far advanced lesions. Once the appropriate sections and fields were identified, the results were consistent among all cases.

The early lesion of PPTB is a post obstructive lobular lipid pneumonia that spreads via bronchi for months prior to caseation [1, 6, 8] (Figure 1). Secreted MTB antigens in the early post-primary phase were detected by immunohistochemistry within alveolar macrophages (Figure 1A), alveolar pneumocytes (Figure 1B), and ciliated bronchiolar epithelial cells (Figure 1C), Alveolar monocytes and macrophages are not necessarily foamy in the early postprimary phase and show only mild expression of MTB antigen compared with the later phases. The mycobacterial antigens were diffusely present throughout the cytoplasm of the cells as is characteristic of secreted antigens rather than the discrete spots characteristic of intact organisms $[8,9]$. PD-L1 expression was present on alveolar pneumocytes, sloughed bronchiolar epithelial cells, alveolar monocytes and macrophages (Figure 2A). PD-1 staining was present on lymphocytes primarily in the interstitium (Figure 2B). COX-2 expression was evident by immunohistochemistry within reactive alveolar pneumocytes (Figure 2C) and bronchiolar epithelial cells (Figure 2D). Alveolar macrophages in the early lesion of PPTB stain with CD163, a marker for M2 polarized macrophages (Figure 2E), Finally, FAS expression was present in the cytoplasmic compartment of reactive alveolar pneumocytes surrounding increasingly foamy alveolar macrophages (Figure 2F). None of these markers were present to any appreciable extent outside of the TB lesions. 


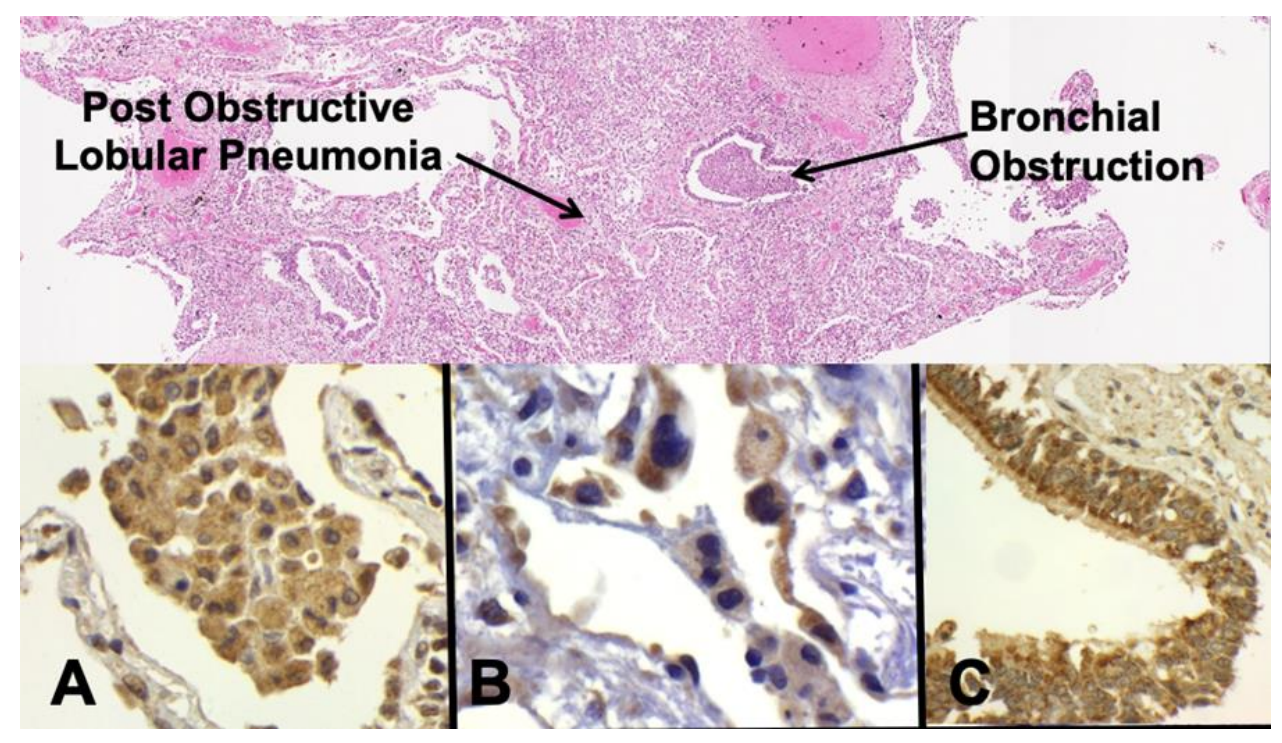

Figure 1. MTB Antigen in the Early Lesion. РРТВ begins as an alveolitis that spreads subclinically as an obstructive lobular pneumonia for 1-2 years before undergoing necrosis as caseous pneumonia to initiate clinical PPTB. Evidence suggests that MTB uses its secreted antigens to direct these lesions towards caseation and cavitation that can transmit infection to new hosts (H\&E stain 20x, partly reproduced from [6]).A. Mycobacterial secreted antigens in alveolar macrophages (Immuno stain 600x).B. Mycobacterial antigens alveolar lining cells types 1 and 2 (Immuno stain 600x).C. Mycobacterial antigens in ciliated bronchial cells (Immuno stain 400x). 


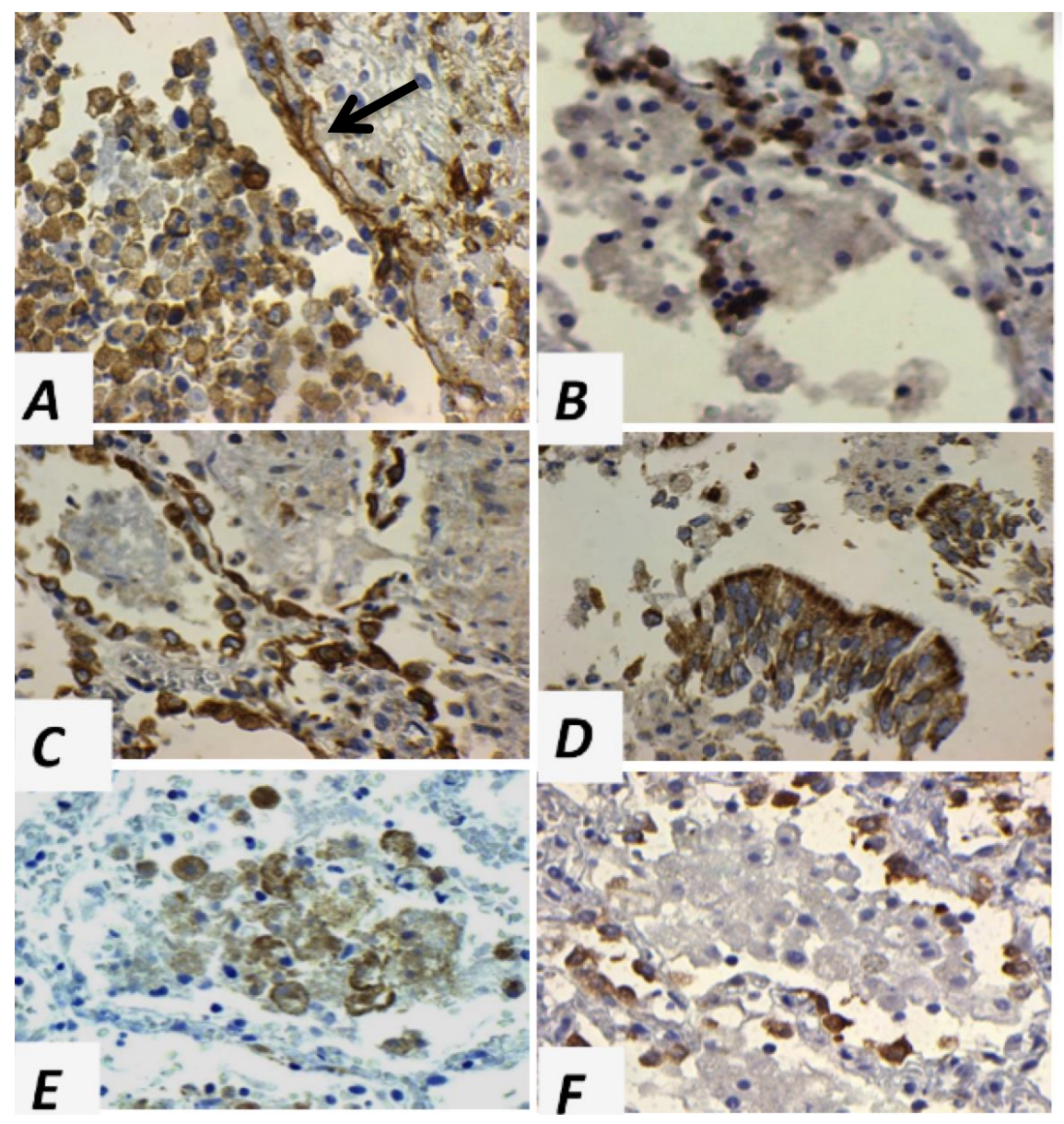

Figure 2. Characterization of the Early Lesion of PPTB. Immunostains for PD-L1, PD-1, COX-2, CD163 and FAS provide evidence that MTB use its secreted antigens to manipulate the host's responses in the early lesion. A. PD-L1 expression on multiple cell types including alveolar pneumocytes (ARROW) and alveolar macrophages (600x). B. PD-1 expression on lymphocytes in adjacent alveolar walls (600x). C. COX-2 in reactive alveolar pneumocytes (600x). D. COX-2 in bronchiolar epithelium (600x). E. CD163 staining of alveolar macrophages marking them as M2 cells (400x). F. FAS expression in the reactive alveolar pneumocytes surrounding the alveolar macrophages (400x).

\section{Discussion}

Since MTB is an obligate human parasite, everything that it does has been selected to ensure survival and transmission among people. MTB secretes is șiover 200 proteins into surrounding media that are required for virulence in humans, but are not necessary for its survival in culture [10]. The functions of some secreted proteins such as ESAT-6 and Ag85 have been studied, but most remain unknown $[9,10]$. The finding of secreted mycobacterial antigens in multiple cell types of the early lesion of PPTB suggests a new synthesis of its pathogenesis.

The early lesion of PPTB is an asymptomatic obstructive lobular pneumonia that develops for 1-2 years before onset of symptoms [11]. It has been observed and described by multiple investigators for over a century, but has never been reported fully developed in any animal model [2-4, 12]. MTB is an obligate human parasite because no animal produces the PPTB lesions that mediate transmission to new hosts. The early lesion is a prolonged sub- 
clinical accumulation of mycobacterial antigens and host lipids in alveolar macrophages, near highly sensitized $\mathrm{T}$ cells with little inflammation even though there may be intense tuberculous inflammation elsewhere in the same lung [8]. The process slowly spreads via bronchi in a process known as bronchogenic TB $[5,13]$.

The finding of MTB antigens in multiple types of cells of the early lesion suggests that MTB use these antigens to coordinate diverse components of the host response to subclinically produce the materials necessary for a massive necrotizing reaction sufficient to form a cavity large enough to support transmission of infection. The components of the early lesion include:

1. Bronchial obstruction traps alveolar macrophages to produce post-obstructive lipid pneumonia [14].

2. Alveolar lining cells use FAS to produce lipids

3. Alveolar macrophages with M2 phenotype (CD163 Staining) become foamy by accumulating host lipids and secreted mycobacterial antigens.

4. Sensitized tissue resident T cells (TRM) accumulate in alveolar walls.

5. PD-L1 expression on alveolar macrophages and alveolar pneumocytes suppress PD$1+\mathrm{T}$ cell activity."

These lesions spread via bronchi as an obstructive lobular pneumonia, recognized by CT scans as the 'tree-in-bud' sign, until they either regress or undergo necrosis to become caseous pneumonia that is either coughed out to form cavities or retained to become the focus of post-primary granulomas [1].

Our new findings include 1) demonstration of mycobacterial antigen within both the type 1 and type 2 alveolar pneumocytes and ciliated bronchiolar lining cells in addition to alveolar monocytes/ macrophages; 2) COX-2 expression in reactive alveolar pneumocytes and ciliated bronchiolar epithelium, 3) expression of fatty FAS that could contribute fatty acids to the foamy alveolar macrophages, and 4) expression of programmed death ligand (PDL)1 on the alveolar monocytes/macrophages, pneumocytes and bronchiolar epithelial cells; and 4) CD163 expression on monocytes/macrophages in alveoli.

In previous studies we reported that the early lesion of PPTB contains foamy alveolar macrophages with the M2 phenotype that marked with CD163, PD-L1, phosphorylated mTOR, insulin-like growth factor-1 receptor (IGF-1R), and human cyclooxygenase 2 (COX2) [15]. Relatively few $\mathrm{CD} 4^{+}$cells were present, but abundant $\mathrm{CD} 8^{+}, \mathrm{PD}-1^{+}$cells were in the alveolar walls. Foamy macrophages stained strongly with CD68 and frequently also with the dendritic cell marker DEC-205. T-regulatory cells were also observed in the early lesion $[16,17]$. These findings suggest that MTB creates a protective microenvironment that accumulates high concentrations of MTB antigens and sensitized $\mathrm{T}$ cells that when released lead to necrosis, cavitation, post-primary granulomas and fibrocaseous disease [17].

The early lesion of PРTB appears to be an attractive target for host directed therapies $[15,18]$. Mice, guinea pigs and rabbits all develop infections that at certain points appear to be models of stages of human PPTB [19]. Evidence for a mechanism of host directed therapy is provided by studies of FAS. The presence of FAS in alveolar lining cells of the early lesion of PPTB indicates increased synthesis of lipids that is a critical component of these lesions. We demonstrated that inclusion a FAS inhibitor, lactoferrin, with a BCG vaccine in mice induced a sustained reduction in lung pathology, but not numbers of organisms in tissue [20]. Metformin and orlistat are also inhibitors of FAS [21-24] and have shown efficacy against TB in preclinical studies of TB [20, 22]. Multiple studies of vitamin D3, metformin, and indomethacin on human specimens in vitro also suggest efficacy against the early lesion. In two separate human trials, metformin treatment was associated with improved control of infection and decreased disease severity [24, 25]. Vitamin D3 has also been shown to reduce ac- 
cumulation of lipids by macrophages and to produce beneficial effects in patients in a controlled trial of pulmonary tuberculosis [26, 27].

Several lines of evidence also suggest that the early lesion might also be an attractive target for vaccines. First, many nascent lesions regress spontaneously. If we knew why, it might be possible to induce all to regress. Second, slowly progressive pulmonary tuberculosis in the mouse is a model of the early lesion of PPTB [28]. Several experimental vaccines have been shown to prevent progression of these lesions [19, 20, 29]. Finally, the M72/AS01E vaccine that was successful in a human clinical trial was designed to induce a strong Th1 macrophage response without a M2 macrophage response [30, 31]. We propose that the protection was provided by suppressing the M2 response and thereby preventing development of the early lesion of PPTB.

\section{Material and Methods}

Formalin-fixed and paraffin-embedded tissue blocks of human tuberculous lung samples were obtained during regular autopsy practice and after the completion of all medical, legal and ethical requirements and were deidentified. Information provided included the age, sex, pulmonary TB as the primary cause of death and negative HIV. Drug sensitivities were not known. Furthermore, this study was conducted according to the principles expressed in the Declaration of Helsinki. Hematoxylin and eosin (H\&E) staining was used to identify blocks with the early lesion of PPTB [1]. Grossly, the early lesion of PPTB may be only a mild thickening of lung tissue. Consequently, it is necessary to prepare multiple sections of lung tissue for microscopic and immuno histochemical examination in order to identify them.

Immunohistochemistry was performed on five micrometer sections that were deparaffinized and stained with monoclonal antibodies conjugated with 3,3'-diaminobenzidine (DAB) for detection by immunohistochemistry. The antibodies used were:" MTB antigen (ab905, Abcam, Cambridge, MA): cyclo-oxygenase (COX)-2 (SP21rabbit, Biocare Medical, Pacheco, CA); Programmed Death-Ligand 1 (PD-L1: Spring Bioscience, Pleasanton, CA); Programmed Death-1 (PD-1, Biocare Medical); and fatty acid synthase (FAS:C20G5, Cell Signaling Technology). Positive and negative controls were run concurrently. The immunohistochemical reactions/ expressions were assessed as weak or strong by visual inspection in comparison with positive and negative controls. All procedures were conducted in a fully accredited clinical laboratory with all relevant positive and negative controls. None of the markers studied are present in appreciable concentrations in normal lung tissue.

\section{Conclusion}

Granulomas are widely considered to be the hallmark of TB. However, the finding of secreted mycobacterial antigens in multiple cells of the early lesions together with markers of multiple immune regulatory pathways suggest that these subclinical asymptomatic lesions are actually the major drivers of clinical PPTB. They accumulate both sensitized $\mathrm{T}$ cells and mycobacterial antigen targets in preparation for necrotizing hypersensitivity reactions. In multiple studies, effective vaccines and host directed therapies caused lessening of the PPTB like pathology [18, 20, 25, 29, 32-34]. Nevertheless, study of these lesions will remain challenging because of the paucity of informative human tissues that can be used both to study the disease and to validate animal models. Fortunately, new multiplex technologies can measure proteins and nucleic acids on slides with a depth and precision undreamed of a decade ago [35]. Such technologies are needed to finally study these critical lesions of tuberculosis and to develop more effective interventions. 
Authors' Contribution: Both authors contributed equally to the writing of the manuscript, and to the review and editing of the final manuscript.

Funding: This research received no external funding.

Conflicts of Interest: The authors declare no conflicts of interest.

\section{References}

1. Hunter RL. Pathology of post primary tuberculosis of the lung: an illustrated critical review. Tuberculosis (Edinb). 2011;91(6):497509. Epub 2011/07/08. doi: 10.1016/j.tube.2011.03.007. PubMed PMID: 21733755; PubMed Central PMCID: PMC3215852.

2. Rich, A. The Pathogenesis of Tuberculosis, Second Edition. Springfield, Illinois: Charles C Thomas; 1951. 1028 p.

3. Canetti, G. The tubercle bacillus in the pulmonary lesion of man. Histobacteriology and its bearing on the therapy of pulmonary tuberculosis. New York: Springer Publishing Compani Inc.; 1955. 226 p.

4. Medlar EM. The pathogenesis of minimal pulmonary tuberculosis; a study of 1,225 necropsies in cases of sudden and unexpected death. Am Rev Tuberc. 1948;58(6):583-611. Epub 1948/12/01. PubMed PMID: 18099839.

5. Im JG, Itoh, H. Tree-in-Bud Pattern of Pulmonary Tuberculosis on Thin-Section CT: Pathological Implications. Korean J Radiol. 2018;19(5):859-65. doi: 10.3348/kjr.2018.19.5.859. PubMed PMID: 30174474; PubMed Central PMCID: PMCPMC6082770.

6. Hunter RL. The Pathogenesis of Tuberculosis-The Koch Phenomenon Reinstated. Pathogens. 2020;9(10):1-25. Epub 2020/10/07. doi: 10.3390/pathogens9100813. PubMed PMID: 33020397.

7. Hunter RL. Tuberculosis as a three-act play: A new paradigm for the pathogenesis of pulmonary tuberculosis. Tuberculosis (Edinb). 2016;97:8-17. doi: 10.1016/j.tube.2015.11.010. PubMed PMID: 26980490; PubMed Central PMCID: PMCPMC4795183.

8. Hunter RL, Actor JK, Hwang SA, Karev, V., Jagannath, C. Pathogenesis of post primary tuberculosis: immunity and hypersensitivity in the development of cavities. Ann Clin Lab Sci. 2014;44(4):365-87. PubMed PMID: 25361920.

9. Mustafa T, Leversen NA, Sviland, L., Wiker HG. Differential in vivo expression of mycobacterial antigens in Mycobacterium tuberculosis infected lungs and lymph node tissues. BMC Infect Dis. 2014;14:535. Epub 2014/10/07. doi: 10.1186/1471-2334-14-535. PubMed PMID: 25284264; PubMed Central PMCID: PMC4287340.

10. Malen H, Berven FS, Fladmark KE, Wiker HG. Comprehensive analysis of exported proteins from Mycobacterium tuberculosis H37Rv. Proteomics. 2007;7(10):1702-18. Epub 2007/04/20. doi: 10.1002/pmic.200600853. PubMed PMID: 17443846.

11. Hunter RL. The Pathogenesis of Tuberculosis: The Early Infiltrate of Post-primary (Adult Pulmonary) Tuberculosis: A Distinct Disease Entity. Front Immunol. 2018;9:2108. Epub 2018/10/05. doi: 10.3389/fimmu.2018.02108. PubMed PMID: 30283448; PubMed Central PMCID: PMCPMC6156532.

12. Basaraba RJ, Hunter RL. Pathology of Tuberculosis: How the Pathology of Human Tuberculosis Informs and Directs Animal Models. Microbiol Spectr. 2017;5(3). doi: 10.1128/microbiolspec.TBTB2-0029-2016. PubMed PMID: 28597826.

13. Medlar EM. Pathogenic concepts of tuberculosis. Am J Medicine. 1950;9:611-22.

14. Hunter RL. On the pathogenesis of post primary tuberculosis: the role of bronchial obstruction in the pathogenesis of cavities. Tuberculosis (Edinb). 2011;91 Suppl 1:S6-10. Epub 2011/11/16. doi: S1472-9792(11)00173-9 [pii] 10.1016/j.tube.2011.10.003. PubMed PMID: 22082617.

15. Brown RE, Hunter RL, Hwang SA. Morphoproteomic-Guided Host-Directed Therapy for Tuberculosis. Front Immunol. 2017;8:78. doi: 10.3389/fimmu.2017.00078. PubMed PMID: 28210262; PubMed Central PMCID: PMCPMC5288338.

16. Welsh KJ, Risin SA, Actor JK, Hunter RL. Immunopathology of postprimary tuberculosis: increased T-regulatory cells and DEC205-positive foamy macrophages in cavitary lesions. Clin Dev Immunol. 2011;2011:307631. Epub 2011/01/05. doi: 10.1155/2011/307631. PubMed PMID: 21197439; PubMed Central PMCID: PMC3010642.

17. Hwang SA, Ali Y, Fedotova E, Hunter RL, Brown RE. Morphoproteomics Identifies the Foamy Alveolar Macrophage as an M2 Phenotype with PD-L1 Expression in the Early Lesion of Post-Primary Tuberculosis: Implications for Host Immune Surveillance and Therapy. Ann Clin Lab Sci. 2020;50(4):429-38. Epub 2020/08/23. PubMed PMID: 32826237.

18. Shim, D., Kim, H., Shin SJ. Mycobacterium tuberculosis Infection-Driven Foamy Macrophages and Their Implications in Tuberculosis Control as Targets for Host-Directed Therapy. Front Immunol. 2020;11:910. Epub 2020/06/02. doi: 10.3389/fimmu.2020.00910. PubMed PMID: 32477367; PubMed Central PMCID: PMCPMC7235167.

19. Hunter, R., Actor, J. The pathogenesis of post-primary tuberculosis. A game changer for vaccine development. Tuberculosis (Edinb). 2019;116S:S114-S7. PubMed PMID: 31076321; PubMed Central PMCID: PMCPMC6626673.

20. Hwang SA, Welsh KJ, Kruzel ML, Actor JK. Lactoferrin Augmentation of the BCG Vaccine Leads to Increased Pulmonary Integrity. Tuberc Res Treat. 2011;2011:835410. doi: 10.1155/2011/835410. PubMed PMID: 22567270; PubMed Central PMCID: PMCPMC3335707.

21. Majumdar A, Wankhade G, Kamble PD, Harinath BC. Effect of HIV protease inhibitors and Orlistat on mycobacterial ES-31 serine protease, a potential drug target in Mycobacterium tuberculosis. Indian J Tuberc. 2011;58(1):4-10. Epub 2011/03/30. PubMed PMID: 21442799. 
22. Wahdan-Alaswad RS, Cochrane DR, Spoelstra NS, Howe EN, Edgerton SM, Anderson SM, et al. Metformin-induced killing of triple-negative breast cancer cells is mediated by reduction in fatty acid synthase via miRNA-193b. Horm Cancer. 2014;5(6):374-89. Epub 2014/09/13. doi: 10.1007/s12672-014-0188-8. PubMed PMID: 25213330; PubMed Central PMCID: PMCPMC4570735.

23. Kridel SJ, Axelrod F, Rozenkrantz N, Smith JW. Orlistat is a novel inhibitor of fatty acid synthase with antitumor activity. Cancer Res. 2004;64(6):2070-5. Epub 2004/03/18. doi: 10.1158/0008-5472.can-03-3645. PubMed PMID: 15026345.

24. Restrepo BI. Metformin: Candidate host-directed therapy for tuberculosis in diabetes and non-diabetes patients. Tuberculosis (Edinb). 2016;101S:S69-S72. Epub 2016/10/11. doi: 10.1016/j.tube.2016.09.008. PubMed PMID: 27720378.

25. Singhal A, Jie L, Kumar P, Hong GS, Leow MK, Paleja, B., et al. Metformin as adjunct antituberculosis therapy. Sci Transl Med. 2014;6(263):263ra159. Epub 2014/11/21. doi: 10.1126/scitranslmed.3009885. PubMed PMID: 25411472.

26. Salamon H, Bruiners N, Lakehal K, Shi L, Ravi J, Yamaguchi KD, et al. Cutting edge: Vitamin D regulates lipid metabolism in Mycobacterium tuberculosis infection. J Immunol. 2014;193(1):30-4. Epub 2014/06/06. doi: 10.4049/jimmunol.1400736. PubMed PMID: 24899504; PubMed Central PMCID: PMCPMC4073889.

27. Mily A, Rekha RS, Kamal SM, Arifuzzaman AS, Rahim, Z., Khan, L., et al. Significant Effects of Oral Phenylbutyrate and Vitamin D3 Adjunctive Therapy in Pulmonary Tuberculosis: A Randomized Controlled Trial. PLoS One. 2015;10(9):e0138340. Epub 2015/09/24. doi: 10.1371/journal.pone.0138340. PubMed PMID: 26394045; PubMed Central PMCID: PMCPMC4578887.

28. Hunter RL, Jagannath, C., Actor JK. Pathology of postprimary tuberculosis in humans and mice: contradiction of long-held beliefs. Tuberculosis (Edinb). 2007;87(4):267-78. Epub 2007/03/21. doi: S1472-9792(06)00120-X [pii] 10.1016/j.tube.2006.11.003. PubMed PMID: 17369095.

29. Liang Y, Bai X, Zhang J, Song J, Yang Y, Yu Q, et al. Ag85A/ESAT-6 chimeric DNA vaccine induces an adverse response in tuberculosis-infected mice. Mol Med Rep. 2016;14(2):1146-52. Epub 2016/06/10. doi: 10.3892/mmr.2016.5364. PubMed PMID: 27279275; PubMed Central PMCID: PMCPMC4940052.

30. Van Der Meeren O, Hatherill M, Nduba V, Wilkinson RJ, Muyoyeta M, Van Brakel E, et al. Phase 2b Controlled Trial of M72/AS01E Vaccine to Prevent Tuberculosis. N Engl J Med. 2018;379(17):1621-34. Epub 2018/10/04. doi: 10.1056/NEJMoa1803484. PubMed PMID: 30280651; PubMed Central PMCID: PMCPMC6151253.

31. Al-Attiyah R, Mustafa AS, Abal AT, El-Shamy AS, Dalemans, W., Skeiky YA. In vitro cellular immune responses to complex and newly defined recombinant antigens of Mycobacterium tuberculosis. Clin Exp Immunol. 2004;138(1):139-44. doi: 10.1111/j.13652249.2004.02609.x. PubMed PMID: 15373916; PubMed Central PMCID: PMCPMC1809193.

32. Okada M, Kita Y, Nakajima T, Kanamaru N, Hashimoto S, Nagasawa, T., et al. Novel prophylactic vaccine using a prime-boost method and hemagglutinating virus of Japan-envelope against tuberculosis. Clin Dev Immunol. 2011;2011:549281. Epub 2011/03/26. doi: 10.1155/2011/549281. PubMed PMID: 21437226; PubMed Central PMCID: PMCPMC3061297.

33. Maurya SK, Aqdas M, Das DK, Singh S, Nadeem, S., Kaur, G., et al. A multiple T cell epitope comprising DNA vaccine boosts the protective efficacy of Bacillus Calmette-Guerin (BCG) against Mycobacterium tuberculosis. BMC Infect Dis. 2020;20(1):677. Epub 2020/09/19. doi: 10.1186/s12879-020-05372-1. PubMed PMID: 32942991; PubMed Central PMCID: PMCPMC7495405.

34. Doimo NT, Zarate-Blades CR, Rodrigues RF, Tefe-Silva C, Trotte MN, Souza PR, et al. Immunotherapy of tuberculosis with Mycobacterium leprae Hsp65 as a DNA vaccine triggers cross-reactive antibodies against mammalian Hsp60 but not pathological autoimmunity. Hum Vaccin Immunother. 2014;10(5):1238-43. Epub 2014/03/13. doi: 10.4161/hv.28249. PubMed PMID: 24607935; PubMed Central PMCID: PMCPMC4896578.

35. Parra ER, Francisco-Cruz A, Wistuba, II. State-of-the-Art of Profiling Immune Contexture in the Era of Multiplexed Staining and Digital Analysis to Study Paraffin Tumor Tissues. Cancers (Basel). 2019;11(2). doi: 10.3390/cancers11020247. PubMed PMID: 30791580; PubMed Central PMCID: PMCPMC6406364. 